\title{
A note on the topographic distortion of magnetotelluric impedance
}

\author{
Filippos Vallianatos \\ Technological Educational Institute of Crete, Branch of Chania, Crete, Greece
}

\begin{abstract}
Magnetotelluric surveys are prone to interpretation errors in the presence of rough topography, which may need to be compensated for. In the present work we assume that the surface of the Earth is simulated by a single-valued, twice differentiable function $f(x, y)$. By appropriately expanding the surface magnetic field, we obtain the distorted magnetotelluric impedance tensor in terms of an expansion depending on the external radii of curvature of $f(x, y)$ at the observation point and on skin depth. Based on first principles, an analytic estimation of the topographic corrections of the magnetotelluric impedance tensor is obtained.
\end{abstract}

Key words magnetotellurics - topography

\section{Introduction}

A problem commonly appearing in magnetotelluric (MT) surveys is topographic distortion (Schnegg et al., 1983, 1986; Chouteau and Bouchard, 1988; Fischer, 1989), especially in the case of measurements carried out in mountainous areas (e.g., Draget et al., 1980; Kurtz et al., 1986; Vallianatos, 1995). Interpretation errors may occur if the distortion is not taken into account, or compensated for. Telluric currents flowing parallel to the surface of the Earth, converge beneath valleys and diverge beneath hills. Therefore the current

Mailing address: Dr. Filippos Vallianatos, Technological Educational Institute of Crete, Department of Natural Resources Engineering, Laboratory of Geophysics and Natural Hazards, 3 Romanou Str., Chalepa, GR-73133 Chania, Crete, Greece; e-mail: fvallian@chania.teiher.gr density $\boldsymbol{J}$ and the associated MT field tend to increase under a valley and decrease under a hill. The reduction of this distortion is therefore an important step toward the correct detection and interpretation of subsurface conductivity distributions. Several studies exist on the effect of topography on MT measurements, most of which adopt a numerical modeling approach (Jiracek, 1973; Harinarayana and Sarma, 1982; Wannamaker et al., 1985, 1986; Jiracek et al., 1986; Xu and Zhou, 1997). Under static conditions, the topographic effects have also been calculated analytically, using SchwarzChristoffel transformations (Thayer, 1975; Harinarayana and Sarma, 1982; Harinarayana, 1986). In the present short work, we present a formulation to analytically estimate the topographic effect, to first and second order correction terms. The proposed approach is based on the simulation of the earth surface by a singlevalued, twice differentiable function and an appropriate expansion of the magnetic field. The estimated corrections depend on frequency and the external radii of curvature of the earth surface at the observation point. 


\section{Mathematical formulation}

As physical basis for the analysis of any magnetotelluric problem, consider Maxwell's equations for an isotropic, source-free region. In the frequency domain, with the constituent relations $\boldsymbol{D}=\varepsilon \boldsymbol{E}, \boldsymbol{B}=\boldsymbol{\mu} \boldsymbol{H}, \boldsymbol{J}=\sigma \boldsymbol{E}$ incorporated, these are (see for example Rokityansky, 1982):

$$
\begin{gathered}
\nabla \times \boldsymbol{H}=(\sigma-i \omega \varepsilon) \boldsymbol{E}=\sigma^{*} \boldsymbol{E} \\
\nabla \times \boldsymbol{E}=i \omega \mu \boldsymbol{H} \\
\nabla \cdot \boldsymbol{H}=0 \quad \text { and } \nabla \cdot \boldsymbol{E}=0 .
\end{gathered}
$$

By combining the curl equations and using the vanishing divergences, it can be seen (Jackson, 1975) that both fields satisfy the homogeneous wave equation (Helmholtz equation) (Wait, 1987)

$$
\nabla^{2}\left[\begin{array}{l}
\boldsymbol{E} \\
\boldsymbol{H}
\end{array}\right]+k^{2}\left[\begin{array}{l}
\boldsymbol{E} \\
\boldsymbol{H}
\end{array}\right]=0
$$

where the propagation constant is $k^{2}=i \omega \mu \sigma^{*}$.

The MT impedance, can be expressed as a rank 2 tensor. If $x$ and $y$ are orthogonal measurement axes on the Earth surface, the tangential to the surface electric field variations $\boldsymbol{E}_{\mu}=(E x, E y)^{T}$ is directly related to the variations of the two magnetic fields $\boldsymbol{H}_{\|}=(H x, H y)^{T}$ by the frequency domain relation

$$
\begin{gathered}
{\left[\begin{array}{c}
E x \\
E y
\end{array}\right]=\left[\begin{array}{cc}
Z x x & Z x y \\
Z y x & Z y y
\end{array}\right]\left[\begin{array}{l}
H x \\
H y
\end{array}\right]} \\
\text { or } \\
\boldsymbol{E}_{/ /}=\overline{\boldsymbol{Z}} \cdot \boldsymbol{H}_{/ /}
\end{gathered}
$$

where $Z_{i j}$ are the elements of the impedance tensor (Kaufman and Keller, 1981).

Next, consider an Earth interface (airEarth interface) described by a profile function $f(x, y)$, which is assumed to be single valued and twice differentiable. The vector normal to the surface is (Lipschutz, 1989; Morse and Feshbach, 1963)

$$
\begin{gathered}
\hat{N}=\Phi(x, y)[\hat{z}-\nabla f(x, y)] \\
\text { where } \\
\Phi(x, y)=\left[1+(\nabla f(x, y))^{2}\right]^{-\frac{1}{2}} .
\end{gathered}
$$

In order to generalize eq. (2.1), we note that

$$
\begin{gathered}
H x=(\hat{\boldsymbol{N}} \times \boldsymbol{H})_{y} \\
\text { and } \\
H y=-(\hat{\boldsymbol{N}} \times \boldsymbol{H})_{x} .
\end{gathered}
$$

Combining eqs. (2.1) and (2.2), gives

$$
\left[\begin{array}{l}
E x \\
E y
\end{array}\right]=\left[\begin{array}{ll}
-Z x y & Z x x \\
-Z y y & Z y x
\end{array}\right]\left[\begin{array}{l}
(\hat{\boldsymbol{N}} \times \boldsymbol{H})_{x} \\
(\hat{\boldsymbol{N}} \times \boldsymbol{H})_{y}
\end{array}\right]
$$

or equivalently

$$
E_{/ l, i}=C_{i j}^{g}(\hat{\boldsymbol{N}} \times \boldsymbol{H})_{j}
$$

where the sum over $j$ is implied (Einstein notation). In the case of a simple one dimensional (1D) structure $\boldsymbol{C}_{i j}^{g}=\boldsymbol{Z}_{o} \boldsymbol{C}_{i j}$ where $C_{i j}$ are the elements of a «normalized» surface impedance tensor. It is straightforward to see that for a flat surface $C_{i j}=\delta_{i j}$. We note that $\boldsymbol{E}_{/ /}$and $\hat{\boldsymbol{N}} \times \boldsymbol{H}$ have only components in the tangent plane, so that the normalized surface impedance tensor $\boldsymbol{C}$, being a surface tensor in curvilinear coordinates, is represented by a $2 \times 2$ matrix. Hereafter, in order to simplify the mathematical formulation, we assume one dimensional structure with a surface profile function $f(x, y)$. 
For $z$ approaching $f(x, y)$ from below, the component of $\boldsymbol{E}$ tangential to the surface is

$$
\begin{gathered}
E_{/ /}=E-\hat{N}(\hat{N} \cdot E) \\
=\frac{1}{\sigma-i \omega \mu}\{\nabla \times \boldsymbol{H}-\hat{\boldsymbol{N}}[\hat{\boldsymbol{N}} \cdot(\nabla \times \boldsymbol{H})]\} .
\end{gathered}
$$

We introduce the coordinate variables $u=x$, $v=y, w=[z-f(x, y)] / D$ where $D(\omega)=i / k(\omega)$, in which the Helmholtz vector equation for $\boldsymbol{H}$ is

$$
\begin{gathered}
{\left[\frac{\partial^{2}}{\partial \boldsymbol{w}^{2}}-\Phi^{2}-\boldsymbol{D} \Phi^{2}\left(\nabla^{2} \boldsymbol{f}+2 \nabla \boldsymbol{f} \cdot \nabla\right) \frac{\partial}{\partial \boldsymbol{w}}+\right.} \\
\left.+\boldsymbol{D}^{2} \Phi^{2}\left(\frac{\partial^{2}}{\partial \boldsymbol{u}^{2}}+\frac{\partial^{2}}{\partial v^{2}}\right)\right] \boldsymbol{H}=0
\end{gathered}
$$

Expanding $\boldsymbol{H}$ in powers of $D(\omega)$ (see Appendix)

$$
\boldsymbol{H}=\sum_{i=0}^{\infty} \boldsymbol{D}^{i} \boldsymbol{h}_{(i)}
$$

we obtain the recursive relation

$$
\begin{gathered}
\left(\frac{\partial^{2}}{\partial \boldsymbol{w}^{2}}-\Phi^{2}\right) \boldsymbol{h}_{(n)}= \\
=\Phi^{2}\left(\nabla^{2} \boldsymbol{f}+2 \nabla \boldsymbol{f} \cdot \nabla\right) \frac{\partial}{\partial \boldsymbol{w}} \boldsymbol{h}_{(n-1)}- \\
-\Phi^{2}\left(\frac{\partial^{2}}{\partial \boldsymbol{u}^{2}}+\frac{\partial^{2}}{\partial v^{2}}\right) \boldsymbol{h}_{(n-2)} .
\end{gathered}
$$

For $n=0$, the solution that vanishes at $\mathrm{Re}$ $(w) \rightarrow-\infty$ is $\boldsymbol{h}_{(0)}=\boldsymbol{a}_{(0)}(u, v) \exp (\Phi w)$. Inserting this in the above recursive relation, we obtain the equation for the next term

$$
\left(\frac{\partial^{2}}{\partial w^{2}}-\Phi^{2}\right) \boldsymbol{h}_{(1)}=(\boldsymbol{W}+\boldsymbol{V} w) \exp (\Phi w) .
$$

With a solution that vanishes at $\operatorname{Re}(w) \rightarrow-\infty$ (Bronson, 1973; Morse and Feshbach, 1963)

$$
\boldsymbol{h}_{(1)}=\left[\boldsymbol{a}_{(1)}+\left(\frac{\boldsymbol{W}}{2 \Phi}-\frac{\boldsymbol{V}}{4 \Phi^{2}}\right) w+\frac{\boldsymbol{V}}{4 \Phi} w^{2}\right] \exp (\Phi w)
$$

where

$$
\begin{gathered}
\boldsymbol{V}=-2 \Phi^{6}(\nabla f \cdot \nabla \nabla f \cdot \nabla f) \boldsymbol{a}_{(0)} \\
\boldsymbol{W}=\Phi^{3}\left[\nabla^{2} f-2 \Phi^{2} \nabla f \cdot \nabla \nabla f \cdot \nabla f+\right. \\
+2(\nabla f \cdot \nabla)] \boldsymbol{a}_{(0)}
\end{gathered}
$$

Continuing in way, we expand the magnetic field in terms that include the expression

$$
\sum_{i} D^{i} \alpha_{(i)}
$$

The arbitrariness of the latter term is partially removed by the remaining Maxwell equation $\nabla \cdot \boldsymbol{H}=0$.

After extensive algebra and differential geometry, we can write

$$
\begin{aligned}
\boldsymbol{E}_{/ /} & =(\sigma-i \omega \varepsilon)^{-1}\left[\frac{\hat{\boldsymbol{N}}}{D} \times \boldsymbol{a}_{(0)}+\hat{\boldsymbol{N}} \times \boldsymbol{a}_{(1)}+\right. \\
& +K_{m} \hat{\boldsymbol{N}} \times \boldsymbol{a}_{(0)}+\nabla \times \boldsymbol{a}_{(0)}+\Phi \hat{\boldsymbol{N}} \times \\
& \left.\times(\nabla \boldsymbol{f} \cdot \nabla) \boldsymbol{a}_{(0)}-\hat{\boldsymbol{N}}\left(\hat{\boldsymbol{N}} \cdot \nabla \times \boldsymbol{a}_{(0)}\right)\right]
\end{aligned}
$$


where

$$
\begin{gathered}
K_{m}=\frac{1}{2}\left(\frac{1}{R_{\min }}+\frac{1}{R_{\max }}\right)=\frac{1}{2} \operatorname{Tr}(S)= \\
=\frac{1}{2} \Phi\left(\nabla^{2} f-\Phi^{2} \nabla f \cdot \nabla \nabla f \cdot \nabla f\right)
\end{gathered}
$$

the mean surface curvature and $S$ is the extrinsic curvature tensor of the surface (Lipschutz, 1989). Finally, by applying vector calculus we obtain at $w=0$

$$
\begin{aligned}
\boldsymbol{E}_{/ /}= & (\sigma-i \omega \varepsilon)^{-1}\left[\left(\frac{1}{D}-K_{m}\right) \hat{\boldsymbol{N}} \times \boldsymbol{H}+\right. \\
& +\Phi \boldsymbol{P} \cdot \nabla \nabla f \cdot(\hat{\boldsymbol{N}} \times \boldsymbol{H})]
\end{aligned}
$$

where $\boldsymbol{P}=\overline{\boldsymbol{I}}-\hat{\boldsymbol{N}} \cdot \hat{\boldsymbol{N}}$ is the projection operator in the tangent plane, which in matrix terms is given as

$$
\boldsymbol{P}=\left(\begin{array}{ccc}
1-\Phi^{2}\left(\frac{\partial^{2} f}{\partial^{2} x}\right)^{2} & -\Phi^{2} \frac{\partial f}{\partial x} \frac{\partial f}{\partial y} & \Phi^{2} \frac{\partial f}{\partial x} \\
-\Phi^{2} \frac{\partial f}{\partial x} \frac{\partial f}{\partial y} & 1-\Phi^{2}\left(\frac{\partial^{2} f}{\partial^{2} y}\right)^{2} & \Phi^{2} \frac{\partial f}{\partial y} \\
\Phi^{2} \frac{\partial f}{\partial x} & \Phi^{2} \frac{\partial f}{\partial y} & 1-\Phi^{2}
\end{array}\right)
$$

Since the operator $\boldsymbol{P}$ acts in the same way as the identity $\overline{\boldsymbol{I}}$ applied on $\hat{\boldsymbol{N}} \times \boldsymbol{H}$, eq. (2.4) could be written as

$$
\begin{gathered}
\boldsymbol{E}_{/ /}= \\
=\frac{1}{\sigma^{*} D}\left[\overline{\boldsymbol{I}}+D\left(\boldsymbol{\Phi} \cdot \nabla \nabla f \cdot \boldsymbol{P}-K_{m} \boldsymbol{P}\right)\right](\hat{\boldsymbol{N}} \times \boldsymbol{H})= \\
=Z_{0}\left[\overline{\boldsymbol{I}}+D\left(\boldsymbol{S}-K_{m} \boldsymbol{P}\right)(\hat{\boldsymbol{N}} \times \boldsymbol{H})\right]
\end{gathered}
$$

where $\boldsymbol{S}=\boldsymbol{\Phi} \boldsymbol{P} \cdot \nabla \nabla f \cdot \boldsymbol{P}$ is the extrinsic curvature tensor of the surface (Lipschutz, 1989). A comparison of eqs. (2.3) and (2.5) leads to the conclusion that the normalized impedance tensor $\boldsymbol{C}=\left(C_{i j}\right)$ has the form $\boldsymbol{C}=\overline{\boldsymbol{I}}+D \boldsymbol{C}^{(1)}$, where $\boldsymbol{C}^{(1)}$ is simply the traceless part of the extrinsic curvature tensor of the surface $S$, given by the expression $\boldsymbol{C}^{(1)}=\boldsymbol{S}-K_{m} \boldsymbol{P}$. We note that the eigenvalues of $\boldsymbol{C}^{(1)}$ are 0 and $\pm \Delta$ where

$$
\Delta=\frac{1}{2}\left(\frac{1}{R_{\min }}-\frac{1}{R_{\max }}\right) .
$$

Since the aforementioned first order correction is expressed by terms that include the second spatial derivatives of $f(x, y)$, it is apparent that the topographic profile with linear slope introduces a topographic correction given by a second order term.

Following the same procedure and keeping second order terms in our approximations, we reach an expression for the normalized impedance tensor

$$
\boldsymbol{C}=\overline{\boldsymbol{I}}+D \boldsymbol{C}^{(1)}-\left(\frac{D^{2}}{4}\right) \overline{\boldsymbol{I}} \operatorname{Tr}\left[\left(C^{(1)}\right)^{2}\right]
$$

where

$$
\begin{gathered}
\operatorname{Tr}\left[\left(C^{(1)}\right)^{2}\right]=2 \Delta^{2}= \\
=\Phi^{2} \operatorname{Tr}\left[(\nabla \nabla f)^{2}\right]-2 \Phi^{4}(\nabla f \cdot \nabla \nabla f)^{2}+ \\
+\Phi^{6}(\nabla f \cdot \nabla \nabla f \cdot \nabla f)^{2}-2 K_{m}^{2}
\end{gathered}
$$

According to the aforementioned mathematical formulation, it is obvious that a crucial parameter in our expansion is the dimensionless product

$$
D(\omega) \frac{\partial^{2} f}{\partial x_{i} \partial x_{j}}
$$


where $x_{1}=x$ and $x_{2}=y$. It is expected that when they are small the retention of only the first few terms in the expansion should yield inaccurate results. Alternatively, we can say that our approximation should be valid as long as $k_{T} D(\omega)$ is much smaller than unity, where $k_{T}$ is the wavenumber that characterizes the roughness of our terrain.

\section{Concluding remarks}

In the present preliminary work we present a formulation to analytically approximate the topographic distortion of the magnetotelluric impedance tensor up to first and second order correction terms. The proposed approach is based on the simulation of the Earth's surface with a singlevalued, twice differentiable function and an appropriate expansion for the magnetic field. The estimated corrections depend on frequency and on the external radii of curvature of the Earth's surface at the observation point, leading to accurate results, as the dimensionless product

$$
D(\omega) \frac{\partial^{2} f}{\partial x_{i} \partial x_{j}} \quad \text { is small. }
$$

The formulation does not make any assumption about the type of topographic profile (i.e. slopes, height). Applying the presented first principles approach, we estimate the correction terms as functions of the lateral second derivatives of the topographic profile. In this way we have obtained a general expression for the topographic correction of the surface MT impedance tensor up to first and second order correction terms. The first order correction $\boldsymbol{C}^{(\mathbf{1})}$ term is given by the traceless part of the extrinsic surface curvature tensor $\boldsymbol{S}$, while the second order correction is just a scalar given in terms of trace of $\boldsymbol{C}^{(1)}$.

\section{Appendix}

Consider a time-harmonic plane wave incident from free space into a homogeneous earth surface described by a terrain function $f(x, y)$. In formulating the problem one assumes that the topography acts as a scatterer. Since the currents flowing parallel to the earth's surface converge beneath valleys and diverge beneath hills, it is straightforward to describe the scattering from the topography by a volume distribution of currents induced inside the «effective topographic scatterer». Maxwell's equations corresponding to this representation of the electric field is (Verdin and Bostick, 1992; Habashy et al., 1993)

$$
\nabla \times \nabla \times \boldsymbol{E}(\boldsymbol{r})-k_{b}^{2} \boldsymbol{E}(\boldsymbol{r})=Q_{T}(\boldsymbol{r}) \boldsymbol{E}(\boldsymbol{r})
$$

where the wavenumber $k_{b}^{2}=i \omega \mu \sigma_{b}$ and the topography factor $Q_{T}(\boldsymbol{r})$ are the terms describing the scattering of currents due to the presence of topography.

The solution to eq. (A.1) can be expressed with a dyadic Green's function which is governed by the appropriate boundary conditions and satisfies the equation

$$
\nabla \times \nabla \times \overline{\boldsymbol{G}}(\boldsymbol{r})-k_{b}^{2} \overline{\boldsymbol{G}}(\boldsymbol{r})=\overline{\boldsymbol{I}} \delta\left(\boldsymbol{r}-\boldsymbol{r}^{\prime}\right)
$$

where $\overline{\boldsymbol{I}}$ is the identity dyadic. For a homogeneous background, the solution to eq. (A.2) is (Kong, 1986)

$\overline{\boldsymbol{G}}\left(\boldsymbol{r}, \boldsymbol{r}^{\prime}\right)=\left[\overline{\boldsymbol{I}}+\frac{1}{k_{b}^{2}} \nabla \nabla\right] g\left(\boldsymbol{r}, \boldsymbol{r}^{\prime}\right)$, where $g\left(r, r^{\prime}\right)$ is the scalar, Green's function which satisfies

$\nabla^{2} g\left(r, r^{\prime}\right)+k_{b}^{2} g\left(r, r^{\prime}\right)=-\delta\left(\boldsymbol{r}-\boldsymbol{r}^{\prime}\right)$ and is given by $g\left(\boldsymbol{r}, \boldsymbol{r}^{\prime}\right)=\frac{e^{i k_{b}\left|\boldsymbol{r}-\boldsymbol{r}^{\prime}\right|}}{4 \pi\left|\boldsymbol{r}-\boldsymbol{r}^{\prime}\right|}=\frac{e^{-\frac{\left|\boldsymbol{r}-\boldsymbol{r}^{\prime}\right|}{D}}}{4 \pi\left|\boldsymbol{r}-\boldsymbol{r}^{\prime}\right|}$. 
Thus the solution to eq. (A.1) is given by (Yaghjlan, 1980; Habashy et al., 1993; Chave and Smith, 1994; Kong, 1986)

$$
\boldsymbol{E}(\boldsymbol{r})=\boldsymbol{E}_{b}(\boldsymbol{r})+\left(\bar{I}+\frac{1}{k_{b}^{2}} \nabla \nabla\right)\left(\int_{V t} d \boldsymbol{r}^{\prime} g\left(\boldsymbol{r}, \boldsymbol{r}^{\prime}\right) Q_{T}\left(\boldsymbol{r}^{\prime}\right) \boldsymbol{E}\left(\boldsymbol{r}^{\prime}\right)\right)
$$

where $V t$ is the effective volume related to the topography and supports the appearance of $Q(r)$. Applying Ampere's law to (A.3) gives an analogous integral equation for the magnetic field

$$
\boldsymbol{H}(\boldsymbol{r})=\boldsymbol{H}_{b}(\boldsymbol{r})+\frac{1}{i \omega \mu} \nabla \times\left(\int_{V t} d \boldsymbol{r}^{\prime} g\left(\boldsymbol{r}, \boldsymbol{r}^{\prime}\right) Q_{T}\left(\boldsymbol{r}^{\prime}\right) \boldsymbol{E}\left(\boldsymbol{r}^{\prime}\right)\right) .
$$

We seek to obtain $\boldsymbol{H}$ as an expansion of $D$. In order to accomplish this we make the following change in variables $x^{\prime}=x+D q_{1}, y^{\prime}=y+D q_{2}$. Introducing the dimensionless variables $q_{1}$ and $q_{2}$ the scalar Green's functions

$$
\begin{aligned}
& g\left(\boldsymbol{r}, \boldsymbol{r}^{\prime}\right)=\frac{e^{-\frac{\left|\boldsymbol{r}-\boldsymbol{r}^{\prime}\right|}{D}}}{4 \pi\left|\boldsymbol{r}-\boldsymbol{r}^{\prime}\right|} \text { where } \\
& R^{2}=\left[\left(x-x^{\prime}\right)^{2}+\left(y-y^{\prime}\right)^{2}+\left(f(x, y)-f\left(x^{\prime}, y^{\prime}\right)\right)^{2}\right]^{1 / 2} \text { possesses the expansion } \\
& g\left(r, r^{\prime}\right)=\frac{1}{D} \frac{e^{-r_{0}}}{r_{0}}\left\{1-\frac{D}{2}\left(\frac{1}{r_{0}}+\frac{1}{r_{0}^{2}}\right) f_{3}\left(q_{1}, q_{2}\right)+\right. \\
& \left.+D^{2}\left[-\frac{1}{2}\left(\frac{1}{r_{0}}+\frac{1}{r_{0}^{2}}\right) g_{4}\left(q_{1}, q_{2}\right)+\frac{1}{8}\left(\frac{1}{r_{0}^{2}}+\frac{1}{r_{0}^{3}}+\frac{1}{r_{0}^{4}}\right) f_{3}^{2}\left(q_{1}, q_{2}\right)+\frac{1}{8}\left(\frac{1}{r_{0}^{3}}+\frac{1}{r_{0}^{4}}\right) h_{4}\left(q_{1}, q_{2}\right)\right]\right\}+O\left(D^{3}\right)= \\
& =\frac{1}{D} \frac{e^{-r_{0}}}{r_{0}}\left(\sum_{n=0}^{\infty} K_{n} D^{n}\right)
\end{aligned}
$$

where (see Morse and Feshbach, 1963; Nietro-Vesperinas, 1991)

$$
r_{0}\left(q_{1}, q_{2}\right)=\left[q_{1}^{2}+q_{2}^{2}+\left(f_{1}(x, y) q_{1}+f_{2}(x, y) q_{2}\right)^{2}\right]^{1 / 2}
$$




$$
\begin{gathered}
f_{1}(x, y)=\partial f / \partial x \quad f_{2}(x, y)=\partial f / \partial y \\
f_{3}\left(q_{1}, q_{2}\right)=\left(f_{i} q_{i}\right)\left(f_{i j} q_{i} q_{j}\right) \\
g_{4}\left(q_{1}, q_{2}\right)=\frac{1}{3}\left(f_{i} q_{i}\right)\left(f_{i j k} q_{i} q_{j} q_{k}\right)+\frac{1}{4}\left(f_{i j} q_{i} q_{j}\right)^{2} \\
h_{4}\left(q_{1}, q_{2}\right)=\left(f_{i} q_{i}\right)^{2}\left(f_{i j} q_{i} q_{j}\right) .
\end{gathered}
$$

When the latter expansion is substituted into eq. (A.5) we obtain

$$
\boldsymbol{H}(\boldsymbol{r})=\boldsymbol{H}_{b}(\boldsymbol{r})+\nabla \times\left(\int_{V t} d \boldsymbol{r}^{\prime} \frac{e^{-r_{0}}}{r_{0}}\left(\sum_{n=0}^{\infty} K_{n} D^{n+1}\right) Q_{T}\left(\boldsymbol{r}^{\prime}\right) \boldsymbol{E}\left(\boldsymbol{r}^{\prime}\right)\right)
$$

From the preceding equation we see that the magnetic field can be expanded in powers of the parameter $D=i / k$ according to $\boldsymbol{H}=\left(\sum_{n=0}^{\infty} \boldsymbol{h}_{(n)} D^{n}\right)$.

\section{REFERENCES}

BRONSON, R. (1973): Introductory Differential Equations (McGraw-Hill), pp. 130.

CHAVE, A.D. and J.T. SMITH (1994): On electric and magnetic galvanic distortion tensor decompositions, J. Geophys. Res., 99, 4669-4682.

Chouteau, M. and K. Bouchard (1988): Two dimensional terrain correction in magnetotelluric surveys, Geophys., 53, 854-862.

Dragert, H., L.K. LAW and P.O. Sule (1980): Magnetotelluric soundings across the Pemberton Volcanic Belt, British Columbia, Can. J. Earth Sci., 17, 161-167.

FISCHER, G. (1989): A strong topographic valley effect in AMT and VLF-R measurements, Geophys. J., 96, 469-475.

HABASHY, T.M., R.W. GROOM and B.R. SPIES (1993): Beyond the Born and Rytov approximations: a nonlinear approach to electromagnetic scattering, J. Geophys. Res., 98, 1759-1775.

HARINARAYANA, T. (1986): Distortion of telluric field measurements near a hill, in 8th Workshop on EM Induction in the Earth and Moon, Neuchatel, Switzerland, pp. 87.

HARINARAYANA, T. and S.V. SARMA (1982): Topographic effects on telluric field measurements, Pageoph, 120, 778-783.

JACKSON, J.D. (1975): Classical Electrodynamics (Wiley), pp. 450.

JIRACEK, G.R. (1973): Numerical comparisons of a modified Rayleigh approach with other rough surface EM scattering solutions, IEEE Trans. Antennas Propagat., AP-21, 393-396.

JIRACEK, G.R., R.P. REDDIG and R.K. KoJIMA (1986): Application of the Rayleigh-FFT technique to magnetotelluric modeling and correction, in 8th Workshop on EM Induction in the Earth and Moon, Neuchatel, Switzerland, pp. 114.

KaufMan, A. A. and G. Keller (1981): The Magnetotelluric Sounding (Elsevier), pp. 470.

KonG, J.A. (1986): Electromagnetic Wave Theory (Wiley), pp. 696.

KuRTZ, R.D., J.M. DELAURIER and J.C. GuPTA (1986): A magnetotelluric sounding across Vancouver Island detects the subducting Juan De Fuca Plate, Nature, 321, 596-599.

LIPSCHUTZ, M.M. (1989): Theory and Problems in Differential Geometry (McGraw-Hill), pp. 263.

Morse, P.M. and H. FESHBACH (1963): Methods of Theoretical Physics, Part II (McGraw-Hill), pp. 1978.

NIETRO-VESPERINAS, M. (1991): Scattering and Diffraction in Physical Optics (John Wiley), pp. 397.

ROKITYANSKI, I.I. (1982): Geoelectromagnetic Investigation of the Earth's Crust and Mantle (Springer-Verlag), pp. 381.

SCHNEGG, P.A., B.V. LE QUANG, G. FISCER and J.T. WEAVER (1983): Audio-magnetotelluric study of a structure with a reverse fault, J. Geomagn. Geoelectr., 35, 653-671.

SCHNEGG, P.A., G. FISCHER, M. BuRKHARD and I. Muller (1986): VLF Studie eines engen Alpentales, Protokoll Kolloquium Elektromagnetische Tiefenforschung, 
Bergisch-Glacbach, Marz, 131-136.

THAYER R.E. (1975): Topographic distortion of telluric currents: a simple calculation, Geophysics, 40, 91-95.

VALLIANATOS, F. (1995): Galvanic distortion of the MTimpedance tensor: Application to Ioannina VAN-station (Greece), Acta Geophys. Pol., 42 (2), 115-127.

VERDIN, C.T. and F.X. BOSTICK (1992): Implications of the Born approximation for the magnetotelluric problem in threedimensional environments, Geophysics, 57, 587-602.

WAIT, J.R. (1987): Electromagnetic Wave Theory (John Wiley \& Sons), pp. 250.

WANNAMAKER, P.E., J.A. STODT and L. RIJO (1985): PW2Dfinite element program for solution of magnetotelluric responses of two-dimensional earth resistivity structure; user documentation, Univ. Utah Res. Inst. Rep. ESL-158, pp. 40. WANNAMAKER, P.E., J.A. STODT and L. RIJo (1986): Twodimensional topographic responses in magnetotellurics modeled using finite elements, Geophysics, 51, 2131-2144.

WANNAMAKER, P.E., J.A. STODT and L. RIJO (1987): A stable finite element solution for two-dimensional magnetotelluric modeling, Geophys. J. R. Astron. Soc., 88, 277-296.

XU, S. and H. ZHOU (1997): Modelling the 2D terrain effect on MT by the boundary-element method, Geophys. Prospect., 45, 931-943.

YAGHJIAN, A.D. (1980): Electric dyadic Green's functions in the source region, Proc. IEEE, 68, 248-263. 